\title{
Analysis of Pesticide Mixtures using Intelligent Biosensors
}

\author{
Montserrat Cortina-Puig, Georges Istamboulie, \\ Thierry Noguer and Jean-Louis Marty \\ Université de Perpignan Via Domitia, IMAGES EA4218
}

France

\section{Introduction}

Pesticides are widely used in agricultural crops, forests and wetlands as insecticides, fungicides, herbicides and nematocides. Many of them are considered to be particularly hazardous compounds and toxic because they inhibit fundamental metabolic pathways.

Due to their high acute toxicity and risk towards the population, some directives have been established to limit the presence of pesticides in water and food resources. Concerning the quality of water for human consumption, the European Council directive 98/83/CE (Drinking Water Directive) has set a maximum admissible concentration of $0.1 \mu \mathrm{g} \mathrm{L}^{-1}$ per pesticide and $0.5 \mu \mathrm{g} \mathrm{L}-1$ for the total amount of pesticides.

Organophosphates (OPs) are a class of synthetic pesticides developed from the Second World War, which are used as insecticides and nerve agents (Bajgar et al., 2004; Raushel, 2002). Since the removal of organochlorine insecticides from use, OPs have become the most widely used insecticides. They are normally used for agricultural, industrial, household and medical purposes. OPs poison insects and mammals by phosphorylation of the acetylcholinesterase (AChE) enzyme at nerve endings (Dubois, 1971; Ecobichon, 2001). Inactivation of this enzyme results in an accumulation of acetylcholine leading to an overstimulation of the effector organ (Aldridge, 1950; Reigart et al., 1999).

The hazardous nature of OPs and their wide usage has led to concerted efforts for developing highly sensitive detection techniques as well as efficient destruction methods for these compounds (Gill et al., 2000). Detection techniques are fundamental in order to accurately determine the level of contamination of waters by pesticides. They are classically based on extraction, cleanup and analysis using gas chromatography (GC) or liquid chromatography (LC) coupled to sensitive and specific detectors (Ballesteros et al., 2004; Geerdink et al., 2002; Kuster et al., 2006; Lacorte et al., 1993). Although they are very sensitive, these techniques are expensive and time consuming (involve extensive preparation steps), they are not adapted for in situ and real time detection and often require highly trained personnel. In addition, these methods are not able to provide any information concerning the toxicity of the sample.

AChE biosensors appear as a rapid and simple alternative method for the detection of OPs insecticides. A successful AChE biosensor for toxicity monitoring should offer comparable

Source: Intelligent and Biosensors, Book edited by: Vernon S. Somerset, ISBN 978-953-7619-58-9, pp. 386, January 2010, INTECH, Croatia, downloaded from SCIYO.COM 
or even better analytical performances than the traditional chromatographic systems. Ideally, such sensors should be small, cheap, simple to handle and able to provide reliable information in real-time without or with a minimum sample preparation.

\section{Acetylcholinesterase-based biosensors}

An alternative to elaborated chromatographic methods is the use of enzymatic determination based on AChE inhibition. Detection kits have been successfully designed based on this principle (Andreescu et al., 2006; No et al., 2007). The most advanced systems described so far are based on the biosensor technology. Numerous sensors have been described for OPs determination based on the inhibition of cholinesterases (ChEs) (Andreescu et al., 2006); some of them involving recombinant AChEs specially tailored to enhance their sensitivity to specific inhibitors (Istamboulie et al., 2007). The mechanism of inhibition of AChE by OP and carbamate compounds is well-known (Aldridge, 1950). The inhibitor phosphorylates or carbamoylates the active site serine and the inhibition can be considered as irreversible in the first 30 min (Boublik et al., 2002).

$$
\begin{aligned}
& K_{d} \quad k_{2} \\
& \mathrm{E}+\mathrm{PX} \leftrightarrow \mathrm{E}^{*} \mathrm{PX} \rightarrow \mathrm{EP}+\mathrm{X}
\end{aligned}
$$

where $\mathrm{E}=$ enzyme, $\mathrm{PX}=$ carbamate or $\mathrm{OP}$ and $\mathrm{X}=$ leaving group.

This scheme can be simplified using the bimolecular constant $k_{i}=k_{2} / K_{d}$ :

$$
\mathrm{E}+\mathrm{PX} \stackrel{k_{i}}{\rightarrow} \mathrm{EP}+\mathrm{X}
$$

Two types of ChEs are known and have been used for designing biosensors: AChE and butyrylcholinesterase (BuChE). BuChE has a similar molecular structure to that of AChE but is characterized by different substrate specificity: AChE preferentially hydrolyses acetyl esters such as acetylcholine, while BuChE hydrolyses butyrylcholine. Another aspect that distinguishes AChE from BuChE is the AChE inhibition by excess of substrate. This property is related to substrate binding and the catalytic mechanism. Apart from the natural substrates, ChEs also hydrolyse esters of thiocholine such as acetylthiocholine, butyrylthiocholine, propionylthiocholine, acetyl- $\beta$-methylthiocholine as well as onitrophenylacetate, indophenylacetate and $\alpha$-naphtyl acetate. Many of these substrates have been used in different $\mathrm{ChE}$ biosensor configurations. AChE enzymes extracted from the Drosophila melanogaster and electric eel are commercially available and are the most widely used for biosensor fabrication. ChEs have been extensively used in biosensor configurations based on amperometric detection. Basically, the first devices described were coupling a ChE with a choline oxidase, the detection being based on either the oxidation of $\mathrm{H}_{2} \mathrm{O}_{2}$ or the reduction of oxygen. This complicated system was further simplified using a synthetic substrate of AChE, acetylthiocholine, which produces under hydrolysis an easily oxidisable compound, thiocholine, according to the following reactions:

$$
\text { Acetylthiocholine } \rightarrow \text { Thiocholine }+\mathrm{CH}_{3} \mathrm{COOH}
$$

2 Thiocholine + Med (ox) $\rightarrow$ Dithiobis(choline) $+2 \mathrm{H}^{+}+$Med (red) 
with Med = electronic mediator,

$$
\text { Med (red) } \rightarrow \text { Med (ox) }+2 \text { e- }
$$

The use of an appropriate mediator, like tetracyanoquinodiemethane (TCNQ) or cobalt phatalocyanine (CoPC) allows decreasing the detection potential to values lower than 100 $\mathrm{mV}$ vs. $\mathrm{Ag} / \mathrm{AgCl}$. The mediator can be used in solution but it is generally incorporated in the working electrode material. The most versatile method for manufacturing the electrode is probably the screen-printing method. This technology allows the production of screenprinted three-electrode system with a low cost and a high reproducibility.

The detection principle of AChE-based biosensors leads on the blocking of thiocholine production by OP insecticides. Typically, amperometric measurements are performed in stirred PBS solution at $\mathrm{pH}$ values comprised between 7 and 8 . After applying the appropriate potential for mediator oxidation, the current intensity is recorded in the presence of a saturating concentration of substrate acetylthiocholine. The time necessary to reach the plateau is $2-3 \mathrm{~min}$. The measured signal corresponds to the difference of current intensity between the baseline and the plateau. The cell is washed with distilled water between measurements. The pesticide detection is made in a three step procedure: first, the initial response of the electrode to acetylthiocholine $(1 \mathrm{mM})$ is recorded two times, then the electrode is incubated in a solution containing a known concentration of insecticide, and finally the residual response of the electrode is recorded again. The percentage of the inhibition is then correlated with the insecticide concentration.

Based on this method, highly sensitive biosensors have been developed in our group using recombinant enzymes and appropriate immobilization methods. We have mainly focused our attention on two insecticides of interest: chlorpyrifos (CPO) and chlorfenvinfos (CFV), which are included in a list of priority substances in the field of water policy (decision 2455/2001/EC) (Istamboulie et al., 2007). The developed sensors allowed the detection of pesticides concentrations as low as $1.310^{-11} \mathrm{M}$ (Istamboulie et al., 2009b).

\section{Artificial neural networks}

One shortcoming in present stage of biosensors development using inhibition of AChE is the fact that various OP and carbamate pesticides inhibit this enzyme to a different extent, rendering calibration for an unknown mixture virtually impossible. To overcome this problem, we have recently described a biosensor associating a highly sensitive geneticallymodified Drosophila melanogaster AChE (B394) with a phosphotriesterase (PTE) (Istamboulie et al., 2009b). This enzyme allows hydrolysing OP compounds with various affinities. The developed device has been shown to allow the discriminative detection of CPO and CFV in a wide range of concentrations. However, the determination of mixtures of pesticides was shown to be impossible without further analysis (Istamboulie et al., 2009b). A detection system capable of discriminating and quantifying several inhibitors in a mixture should provide a more reliable and robust biosensor analysis. In this sense, the use of a sensor array coupled with a chemometric tool, such as an Artificial Neural Network (ANN) employed for data treatment, could substantially improve biosensor selectivity and allow exact identification of the inhibitor present in a sample (Bachmann et al., 2000; Bachmann et al., 1999). 
An ANN is a systematic procedure of data processing inspired by the nervous system function in animals. It tries to reproduce the brain logical operation using a collection of neuron-like entities to perform processing of input data (Cartwright, 1993).

The basic processing unit of an ANN is called perceptron (Svozil et al., 1997), which is a crude approximation to the biological neuron, the cell in the nervous system. It is a decisionmaking unit with several input connections and a single output, as shown in Figure 1. A signal $p_{i}$ which is delivered from an input $i$ is multiplied on arrival by a connection weight $w_{i}$, so that each signal appears at the perceptron as the weighted value $w_{i} p_{i}$. The perceptron sums the incoming signals and adds a bias $b$ to give a total signal $n$. To this sum, a transfer function, usually a step-function, is applied to produce the output $a$. Inspired on its physiology, if the sum of inputs reaches the threshold level, the neuron is turned "on" and a message is sent out. If the sum is below the threshold value, the neuron is quiescent and remains "off". This process is summarized in Equation 1.

$$
a=f\left(b+\sum_{j=1}^{i} w_{i} p_{i}\right)
$$

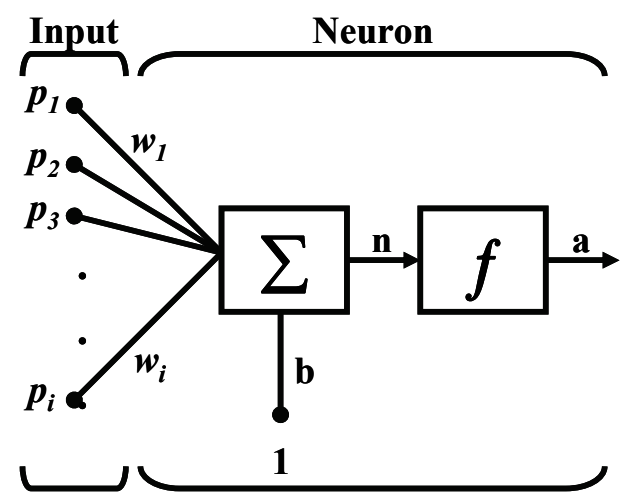

Fig. 1. Schematic representation of the perceptron

A unique condition must be fulfilled: the problem has to be linearly separable. However, most significant scientific problems are not. The failure of the perceptron to solve real-world scientific problems highlights the rather resemblance between it and the brain. The rich network of neurons that makes the brain suggested that a promising step would be to add more perceptrons. This can be done in two different ways: first giving the perceptrons neighbours to form a layer of units which share inputs from the environment; and secondly by introducing further layers, each taking as their input, the output from the previous layer. In this way, the most common ANN used for numerical models is known as the multilayer feedforward network, and is shown in Figure 2.

The path of the departure information begins entering an input layer, whose purpose is just to distribute incoming signals to the next layer; it does not perform any thresholding, thus the units are not perceptrons in its right sense. The perceptrons in the second layer constitute a hidden layer since they communicate with the environment only by sending or 
receiving messages to units in the layers to which they are connected. The output layer provides a link between the artificial network and the outside world, submitting the processed information. Every perceptron is connected to all units in the adjoining layers, but there are no connections between units in the same layer. For this reason it is called a fullyconnected layered feedforward.

As can be seen in Figure 2, all units have at least one input and one output. Their output may consist of the sum of their inputs but usually a transfer function is applied to this sum. Actually, the non-linear modelling capabilities arise because of these transfer functions. In the hidden layer, sigmoid functions are often used, whereas in the output layers, linear functions are used in quantification problems. Some of the transfer functions that can be used are shown in Figure 3.

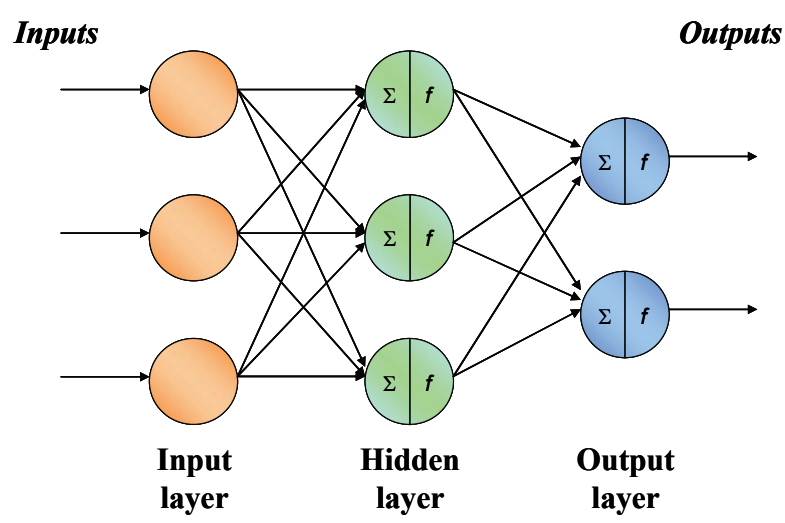

Fig. 2. Schematic structure of an ANN

(a)

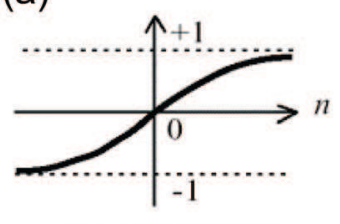

$a=\operatorname{tansig}(n)$ (b)

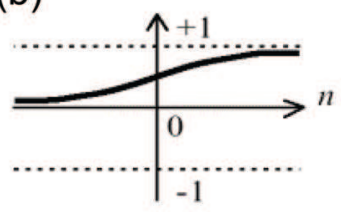

$a=\log \operatorname{sig}(n)$ (c) $a$

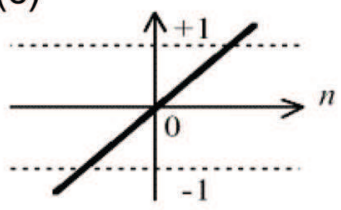

$a=\operatorname{purelin}(n)$

Fig. 3. Representation of three commonly used transfer functions: tan-sigmoid (a), logsigmoid (b) and linear (c)

The output of a unit is sent with an attenuation factor (weight) to a unit in the next layer. These weights are randomly initialized before training. The model is built by repeatedly showing training instances (samples) to the network and adapting the weights so that the 
difference between the output units and the target values is minimized. Usually, the complete training set should be offered many times before a reasonable model is obtained. One pass of the randomly ordered instances in the training set is called an epoch. A vast number of different training algorithms exist (Rumelhart et al., 1986). The most well-known is called the back-propagation learning rule, whose objective is to adjust connection weights in a fashion which reduces the error function $E_{p}$ (Equation 2):

$$
E_{p}=\frac{1}{2} \sum\left(t_{p j}-o_{p j}\right)^{2}
$$

where $o_{p j}$ is the certain instant output and $t_{p j}$ is the target output, for each neuron $j$ and each set training member $p$.

A way to accomplish this is by using the gradient-descent algorithm, an iterative optimisation procedure in which the connection weights are adjusted in a fashion which reduces the error most rapidly, by moving the system downwards in the direction of maximum gradient (Bishop, 1995). The weight of a connection at stage $(t+1)$ of the training is related to its weight at stage $(t)$ by the Equation 3 :

$$
w_{i j}(t+1)=w_{i j}(t)+\alpha \cdot \delta_{p j} o_{p j}
$$

where $\alpha$ is a gain term, known as the training rate factor, $\delta$ is the size of change and the product $\delta_{p j} \cdot o_{p j}$ represents the gradient contribution. The training rate factor varies between 0 and 1 and accelerates or slows down the descent towards the global minimum of the system. It is possible to derive expressions prescribing the size of the changes that must be made at the connection weights to reduce the error signal (Rumelhart et al., 1986).

For the output layer:

$$
\delta_{p j}=k o_{p j}\left(1-o_{p j}\right)\left(t_{p j}-o_{p j}\right)
$$

For the hidden layer:

$$
\delta_{p j}=k o_{p j}\left(1-o_{p j}\right) \sum_{k} \delta_{p k} w_{j k}
$$

These expressions, which are known as the generalized delta rule, show that the extent of the adjustment of connection weights to hidden layers depends upon errors in the subsequent layers, so modifications are made first to the output layer weights, and then the error is then propagated successively back through the hidden layers - this is referred to as backpropagation (of error). Each unit receives an amount of the error signal which is in proportion to its contribution to the output signal, and the connection weights are adjusted by an amount proportional to this error.

Backpropagation by gradient descent is generally a reliable procedure; nevertheless, it has its limitations: it is not a fast training method and it can be trapped in local minima. To avoid the latter, a variant of the above algorithm called gradient-descent with momentum (GDM) introduces a third term, $\beta$ :

$$
w_{i j}(t+1)=w_{i j}(t)+\alpha \cdot \delta_{p j} o_{p j}+\beta \Delta w_{i j}(t)
$$


The term $\beta$, referred to as the momentum, takes a fixed value between 0 and 1 and serves to reduce to the probability of the system being trapped in a local minimum.

A more efficient minimization algorithms is the Levenberg-Marquardt (LM) (Demuth et al., 1992; Rao, 1984), which is between 10 and 100 times faster than gradient-descent, since it employs a second-derivative approach, while GDM employs only first-derivative terms. As the calculation of the Hessian matrix (matrix of the second derivatives of the error in respect of the weights) is a very cumbersome task, LM algorithm employs an approximation starting with a Jacobian matrix (matrix of the first derivatives of the error in respect of the weights), since it is much easier to calculate the Jacobian than the Hessian matrix (Levenberg, 1944; Marquardt, 1963). Therefore, the weights can be calculated as:

$$
w_{i j}(t+1)=w_{i j}(t)-\left[J^{T} J+\mu I\right]^{-1} J^{T} e
$$

where $J$ stands for the Jacobian matrix, $\mu$ for an adjustment factor, $I$ for the identity matrix and $e$ for a vector of network errors. When $\mu$ is large, this becomes gradient descent with a small step size. Thus, the aim is to keep $\mu$ as small as possible. This way, if $\mu$ is decreased after every epoch, this becomes a very effective algorithm (Demuth et al., 1992).

One of the problems that may occur during neural network training is called overfitting (Freeman et al., 1991; Svozil et al., 1997). This situation occurs when the error on the training set is driven to a very small value, but when new data is presented to the network the error is large. Two different methods can be used to avoid overfitting:

1. Bayesian Regularization (BR): This technique searches for the simplest network which adjusts itself to the function to be approximated, but which also is able to predict most efficiently the points that did not participate in the training (Mackay, 1995). In contrast to gradient descent, in this case not only the global error of the ANN is taken into consideration, but also the value of every single weight of the network. Therefore, the values of the weights are minimized, and the network's complexity is reduced, the responses are smoothened and overfitting is avoided. Furthermore, certain neurons are pruned if all its weights are equal to zero.

2. Early stopping: This technique employs additional data to avoid the undesired overfitting. In this case the available data is divided into three subsets. The first subset is the training set, which is used for computing the gradient and updating the network weights and biases, viz. to accomplish learning of the ANN. The second subset is the validation set, which is used during the training process to check the trend presented by this error from data not used for training. The validation error will normally decrease during the initial phase of training, as does the training set error. However, when the network begins to overfit the data, the error on the validation set will typically begin to rise. When the validation error increases for a specified number of iterations, the training is stopped, and the weights and biases at the minimum of the validation error are returned. The test set error is a third subset, not used at all during the training process or its internal monitoring, but it is used to compare performance of different models. If the error in this external test set reaches a minimum at a significantly different iteration number than the validation set error, this may indicate a poor division of the data set. 


\section{Multicomponent determination of pesticides based on enzymatic inhibition}

Several intelligent biosensors for the resolution of mixtures of pesticides have been developed based on the principle of the AChE inhibition and chemometric data analysis using ANNs.

Bachmann et Schmid (Bachmann et al., 1999) developed a sensitive screen-printed amperometric multielectrode biosensor for the rapid discrimination of the insecticides paraoxon and carbofuran in mixtures. For this purpose, four types of native or recombinant AChEs (Electric eel, bovine erythrocytes, rat brain and Drosophila melanogaster) were immobilized by screen printing on four-electrode thick film sensors in sets containing each AChE. The sensors registered a detection range for both analytes of $0.2-20 \mu \mathrm{g} \mathrm{L}^{-1}$ with an overall assay time of less than $60 \mathrm{~min}$. The individual inhibition pattern of each AChEanalyte combination enabled the discrimination of both analytes by the use of ANNs. Thus, paraoxon and carbofuran in mixtures displaying a concentration range of $0-20 \mu \mathrm{g} \mathrm{L}^{-1}$ for each analyte could be analysed with prediction errors of $0.9 \mu \mathrm{g} \mathrm{L}^{-1}$ for paraoxon and $1.4 \mathrm{~L}^{-1}$ for carbofuran.

The same group improved the multianalyte detection by selecting different AChE mutants (Bachmann et al., 2000). They developed two different multisensors: the first one included the wild-type Drosophila AChE and mutants Y408F, F368L and F368H; in the second one, the use of the mutant F368W instead of the F368H increased the sensor's capacity even further. Both multisensors were used for inhibition analysis of binary paraoxon and carbofuran mixtures in a concentration range $0-5 \mu \mathrm{g} \mathrm{L}-1$, followed by data analysis using feedforward ANNs. The two analytes were determined with prediction errors of $0.4 \mu \mathrm{g} \mathrm{L}-1$ for paraoxon and $0.5 \mu \mathrm{g} \mathrm{\textrm {L } ^ { - 1 }}$ for carbofuran. A complete biosensor assay and subsequent ANN evaluation was completed within $40 \mathrm{~min}$. In addition, the second multisensor was also investigated for analyte discrimination in real water samples. Finally, the properties of the multisensors were confirmed by simultaneous detection of binary OP mixtures. Malaoxon and paraoxon in composite solutions of $0-5 \mu \mathrm{g} \mathrm{\textrm {L } ^ { - 1 }}$ were discriminated with predication errors of 0.9 and $1.6 \mu \mathrm{g} \mathrm{L-1}$, respectively.

Our group has developed different amperometric systems to resolve pesticide mixtures (Cortina et al., 2008; Istamboulie et al., 2009a; Valdés-Ramírez et al., 2009). These systems have been termed as electronic tongues, since they combine a sensor array to generate multidimensional data and their proper processing to obtain more detailed information (Holmberg et al., 2004). The combined response of these electrodes was always modelled by means of ANNs.

Firstly, an electronic tongue to quantify dichlorvos and carbofuran pesticide mixtures was developed (Cortina et al., 2008). In that case, the signal was generated from a three biosensor array that used different AChE enzymes: the wild type from Electric eel and two different genetically modified enzymes (B1 and B394). Mean values of concentration of evaluated pesticides were $0.79 \mathrm{nM}$ for dichlorvos and $4.1 \mathrm{nM}$ for carbofuran. The developed electronic tongue was also applied to the determination of dichlorvos and carbofuran in real water samples. Both pesticides could be determined with low errors from a direct measurement step.

Secondly, a bioelectronic tongue was developed to resolve pesticide mixtures of two OP pesticides: dichlorvos and methylparaoxon (Valdés-Ramírez et al., 2009). The biosensor 
array also used three different AChE enzymes: the wild type from Electric eel and two different genetically modified enzymes, B1 and B394 mutants, from Drosophila melanogaster. In this case, the biosensor array was used in a flow injection system, permitting to perform automatically the inhibition assay of the pesticide mixture. The inhibition response triplet was trained with mixture solutions that contained dichlorvos from $10^{-4}$ to $0.1 \mu \mathrm{M}$ and methylparaoxon from 0.001 to $2.5 \mu \mathrm{M}$. When applied to real samples, the two pesticides could be determined with low errors using an extremely simple procedure.

Finally, an amperometric AChE biosensor array was developed to resolve mixtures of two OP insecticides: CPO and CFV (Istamboulie et al., 2009a). Three different biosensors were built using the wild type from Electric eel, the genetically modified Drosophila melanogaster AChE B394 and B394 co-immobilized with a PTE. Specifically two different ANNs were constructed. The first one was used to model the combined response of B394 + PTE and Electric eel biosensors and was applied when the concentration of CPO was high and the other, modelling the combined response of B394 + PTE and B394 biosensors, was applied with low concentrations of CPO. In both cases, good prediction ability was obtained. The developed system was also applied to the determination of CPO and CFV pesticides in real water samples. Both pesticides could be quantified with low errors from a direct measurement step.

\section{Conclusions}

Acetyl- and butyl-cholinesterases have been described for many years as sensitive tools for the detection of many neurotoxic compounds such as insecticides (OPs and carbamates), chemical weapons and toxins (anatoxin-a(s)). They have been extensively used in biosensor configurations based on amperometric detection. Basically, the first devices described were coupling a cholinesterase with a choline oxidase, the detection being based on either the oxidation of $\mathrm{H}_{2} \mathrm{O}_{2}$ or the reduction of oxygen. This complicated system was further simplified using a synthetic substrate of $\mathrm{AChE}$, acetylthiocholine, which produces under hydrolysis an easily oxidisable compound, thiocholine. Since then, the sensitivity of AChEbased sensors has been greatly improved, mainly due to the use of genetically modified AChEs, which were specifically modified for their sensitivity to special classes of inhibitors. However, the described devices often lack of selectivity and specificity, mainly due to the fact that AChE enzymes are globally sensitive to a class of inhibitors. The selectivity of AChE-based sensors can be tuned by the use of PTE, an enzyme hydrolysing specifically some OP compounds. This enzyme has been successfully coupled to AChE for designing sensors selective to two OP compounds of great environmental concern: CPO and CFV. Despite this progress, the main problem still remained the analysis of pesticide mixtures, which can be solved in some cases by the use of a sensor array coupled with a chemometric tool. In this sense ANNs have been found to be powerful tools, particularly suited for various tasks in information processing. ANNs are non-parametric calibration methods specially created to process non-linear information. It has been demonstrated that by combining native and recombinant variant of AChE with ANNs data processing, a sensitive multianalyte detection is possible. 


\section{References}

Aldridge, W.N. (1950). Some properties of specific cholinesterase with particular reference to the mechanism of inhibition by diethyl p-nitrophenyl thiophosphate (E 605) and analogues. Biochemical Journal, 46, 451-60

Andreescu, S. \& Marty, J.-L. (2006). Twenty years research in cholinesterase biosensors: From basic research to practical applications. Biomolecular Engineering, 23, $1-15$

Bachmann, T.T., Leca, B., Vilatte, F., Marty, J.-L., Fournier, D. \& Schmid, R.D. (2000). Improved multianalyte detection of organophosphates and carbamates with disposable multielectrode biosensors using recombinant mutants of Drosophila acetylcholinesterase and artificial neural networks. Biosensors and Bioelectronics, 15, 193-201

Bachmann, T.T. \& Schmid, R.D. (1999). A disposable multielectrode biosensor for rapid simultaneous detection of the insecticides paraoxon and carbofuran at high resolution. Analytica Chimica Acta, 401, 95-103

Bajgar, J. \& Gregory, S.M. (2004). Organophosphates/nerve agent poisoning: Mechanism of action, diagnosis, prophylaxis, and treatment. Advances in Clinical Chemistry, Volume 38, 151-216

Ballesteros, E. \& Parrado, M.J. (2004). Continuous solid-phase extraction and gas chromatographic determination of organophosphorus pesticides in natural and drinking waters. Journal of Chromatography A, 1029, 267-73

Bishop, C.M. (1995). Neural Networks for Pattern Recognition, Oxford University Press, 0-19853864-2, Oxford

Boublik, Y., Saint-Aguet, P., Lougarre, A., Arnaud, M., Villatte, F., Estrada-Mondaca, S. \& Fournier, D. (2002). Acetylcholinesterase engineering for detection of insecticide residues. Protein Engineering, 15, 43-50

Cartwright, H.M. (1993). Applications of artificial intelligence in chemistry, Oxford University Press, 0-19-855736-1, New York

Cortina, M., Del Valle, M. \& Marty, J.-L. (2008). Electronic Tongue Using an Enzyme Inhibition Biosensor Array for the Resolution of Pesticide Mixtures. Electroanalysis, 20, 54-60

Demuth, H. \& Beale, M. (1992). Neural Network Toolbox, for Use with MATLAB, Mathworks Inc, Natick, MA, USA

Dubois, K.P. (1971). The toxicity of organophosphorous compounds to mammals. Bulletin of the World Health Organization, 44, 233-40

Ecobichon, D.J. (2001). Toxic effects of pesticides. In: Casarett $\mathcal{E}$ Doull's Toxicology: The Basic Science of Poisons, Klaassen, C. (Ed.), 763-810, Mc Graw-Hill, New York

Freeman, J.A. \& Skapura, D.M. (1991). Neural Networks: Algorithms, Applications and Programming Techniques, Addison-Wesley, 0-20-151376-5, Reading, MA, USA

Geerdink, R.B., Niessen, W.M.A. \& Brinkman, U.A.T. (2002). Trace-level determination of pesticides in water by means of liquid and gas chromatography. Journal of Chromatography A, 970, 65-93

Gill, I. \& Ballesteros, A. (2000). Degradation of organophosphorous nerve agents by enzyme-polymer nanocomposites: Efficient biocatalytic materials for personal 
protection and large-scale detoxification. Biotechnology and Bioengineering, 70, 400-10

Holmberg, M., Eriksson, M., Krantz-Rulcker, C., Artursson, T., Winquist, F., Lloyd-Spetz, A. \& Lundstrom, I. (2004). 2nd Workshop of the Second Network on Artificial Olfactory Sensing (NOSE II). Sensors and Actuators B: Chemical, 101, 213-23

Istamboulie, G., Andreescu, S., Marty, J.-L. \& Noguer, T. (2007). Highly sensitive detection of organophosphorus insecticides using magnetic microbeads and genetically engineered acetylcholinesterase. Biosensors and Bioelectronics, 23, 506-12

Istamboulie, G., Cortina-Puig, M., Marty, J.L. \& Noguer, T. (2009a). The use of Artificial Neural Networks for the selective detection of two organophosphate insecticides: Chlorpyrifos and chlorfenvinfos. Talanta, 79, 507-11

Istamboulie, G., Fournier, D., Marty, J.-L. \& Noguer, T. (2009b). Phosphotriesterase: A complementary tool for the selective detection of two organophosphate insecticides: Chlorpyrifos and chlorfenvinfos. Talanta, 77, 1627-31

Kuster, M., López De Alda, M. \& Barceló, D. (2006). Analysis of pesticides in water by liquid chromatography-tandem mass spectrometric techniques. Mass Spectrometry Reviews, 25, 900-16

Lacorte, S., Molina, C. \& Barceló, D. (1993). Screening of organophosphorus pesticides in environmental matrices by various gas chromatographic techniques. Analytica Chimica Acta, 281, 71-84

Levenberg, K. (1944). Method for the Solution of Certain Problems in Least Squares. Quarterly of Applied Mathematics, 2, 164-68

Mackay, J.C. (1995). Probable networks and plausible predictions: a review of practical Bayesian methods for supervised neural networks. Network: Computation in Neural Systems, 6, 469-505

Marquardt, D. (1963). An algorithm for least-squares estimation of nonlinear parameters. SIAM Journal on Applied Mathematics, 11, 431-41

No, H.-Y., Kim, Y.A., Lee, Y.T. \& Lee, H.-S. (2007). Cholinesterase-based dipstick assay for the detection of organophosphate and carbamate pesticides. Analytica Chimica Acta, $594,37-43$

Rao, S.S. (1984). Optimization: Theory and applications, Halsted Press, 0-47-027483-2, New York

Raushel, F.M. (2002). Bacterial detoxification of organophosphate nerve agents. Current Opinion in Microbiology, 5, 288-95

Reigart, R. \& Roberts, J. (Eds.) (1999). Recognition and Management of Pesticide Poisonings, U.S. Environmental Protection Agency, USA

Rumelhart, D.E., Hinton, G.E. \& Williams, R.J. (1986). Parallel Distributed Processing: Explorations in the Microstructure of Cognition (Vol 1: Foundations). In: Learning internal representations by error propagation, Rumelhart, D.E. \& Mcclelland, J.L. (Eds.), MIT Press, 0-262-68053-X, London

Svozil, D., Kvasnick, V. \& Pospichal, J. (1997). Introduction to multi-layer feed-forward neural networks. Chemometrics and Intelligent Laboratory Systems, 39, 43-62 
Valdés-Ramírez, G., Gutiérrez, M., Del Valle, M., Ramírez-Silva, M.T., Fournier, D. \& Marty, J.L. (2009). Automated resolution of dichlorvos and methylparaoxon pesticide mixtures employing a Flow Injection system with an inhibition electronic tongue. Biosensors and Bioelectronics, 24, 1103-08 


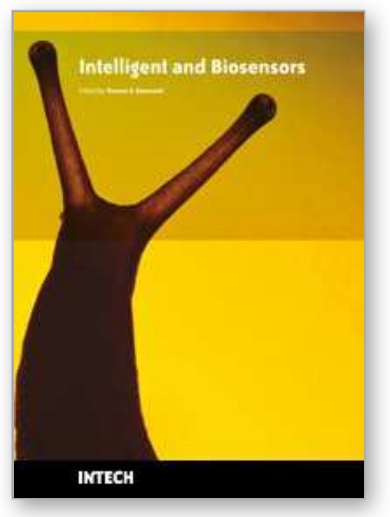

\author{
Intelligent and Biosensors \\ Edited by Vernon S. Somerset
}

ISBN 978-953-7619-58-9

Hard cover, 386 pages

Publisher InTech

Published online 01, January, 2010

Published in print edition January, 2010

The use of intelligent sensors have revolutionized the way in which we gather data from the world around us, how we extract useful information from that data, and the manner in which we use the newly obtained information for various operations and decision making. This book is an attempt to highlight the current research in the field of Intelligent and Biosensors, thereby describing state-of-the-art techniques in the field and emerging new technologies, also showcasing some examples and applications.

\title{
How to reference
}

In order to correctly reference this scholarly work, feel free to copy and paste the following:

Montserrat Cortina-Puig, Georges Istamboulie, Thierry Noguer and Jean-Louis Marty (2010). Analysis of Pesticide Mixtures Using Intelligent Biosensors, Intelligent and Biosensors, Vernon S. Somerset (Ed.), ISBN: 978-953-7619-58-9, InTech, Available from: http://www.intechopen.com/books/intelligent-andbiosensors/analysis-of-pesticide-mixtures-using-intelligent-biosensors

\section{INTECH}

open science | open minds

\section{InTech Europe}

University Campus STeP Ri

Slavka Krautzeka 83/A

51000 Rijeka, Croatia

Phone: +385 (51) 770447

Fax: +385 (51) 686166

www.intechopen.com

\section{InTech China}

Unit 405, Office Block, Hotel Equatorial Shanghai

No.65, Yan An Road (West), Shanghai, 200040, China

中国上海市延安西路65号上海国际贵都大饭店办公楼 405 单元

Phone: +86-21-62489820

Fax: $+86-21-62489821$ 
(C) 2010 The Author(s). Licensee IntechOpen. This chapter is distributed under the terms of the Creative Commons Attribution-NonCommercialShareAlike-3.0 License, which permits use, distribution and reproduction for non-commercial purposes, provided the original is properly cited and derivative works building on this content are distributed under the same license. 